\title{
UN AÑO DE CLAROSCUROS PARA LA ISLA: TAIWÁN EN $2018^{1}$
}

Fabricio Antonio Fonseca Fernández

Universidad de Tamkang

\section{INTRODUCCIÓN}

En el presente capítulo se realiza un breve recorrido por los acontecimientos más importantes relacionados con Taiwán durante 2018. Después de la arrasadora victoria de Tsai Ing-wen

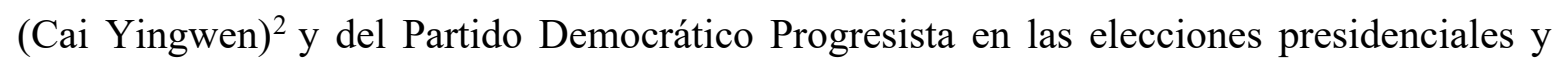
legislativas de 2016, las elecciones locales intermedias fueron vistas aún más de dos años después como un referendo en el desempeño del gobierno. De igual modo, otros hechos marcaron el desarrollo de diferentes aspectos socioeconómicos y sociopolíticos en la isla.

Ahora bien, en la primera sección se presenta un repaso de los sucesos relacionados con la política interna en Taiwán, así como algunos datos importantes en materia económica y de comercio. La segunda sección está dedicada al desarrollo de las relaciones entre uno y otro lados del estrecho de Taiwán en 2018, año que quedó marcado por la persistente falta de diálogo entre ambas partes, resultado de la negativa del gobierno de Tsai para reconocer el Consenso de 1992, así como de un incremento en las presiones por parte de Beijing, que fueron más allá del ámbito político, pues se expandieron al mundo de los negocios. El

${ }^{1}$ Este capítulo fue escrito antes de las elecciones de noviembre de 2018.

${ }^{2}$ Nota sobre los nombres: En este capítulo preservo los nombres de personas y lugares de Taiwán como aparecen comúnmente en la prensa y documentos académicos de lengua extranjera en la isla, para facilitar su identificación. A diferencia de China continental, Taiwán carece de uniformidad a la hora de romanizar los nombres propios, por lo que se recurre a diferentes sistemas que hacen difícil su correcta lectura. Por esa razón, junto a los nombres he agregado la forma como deben leerse los caracteres chinos usando el sistema Hanyu Pinyin, prevaleciente entre las personas que en la actualidad estudian el mandarín. 
empeoramiento de las relaciones entre ambos lados también se debió a las crecientes tensiones entre los gobiernos de Estados Unidos y la República Popular China (RPCh). Por esta razón, en la tercera sección se repasan los acontecimientos más relevantes en las relaciones exteriores de la isla, particularmente por su creciente acercamiento con Washington, así como con otros países en su entorno. Esta parte también analiza las dificultades experimentadas en la isla para mantener su espacio internacional, en particular la pérdida de contacto con países con quienes mantuvo relaciones diplomáticas hasta ese año, hecho que prendió las alertas no sólo en Taipéi, sino también en las oficinas del Departamento de Estado y el Capitolio estadounidenses. Finalmente, algunas consideraciones son presentadas en la última sección.

\section{LA POLÍTICA INTERNA Y LA SITUACIÓN ECONÓMICA Y SOCIAL DE LA ISLA}

\section{Los retos económicos y los desastres naturales}

La madrugada del 6 de febrero de 2018 un terremoto de magnitud 6.4 tuvo lugar en las costas orientales de Taiwán, con epicentro cerca de la ciudad de Hualien (Hualian). Este terremoto ocasionó la muerte de 17 personas tras el colapso de algunos edificios, en particular un hotel de varios pisos, más centenares de heridos y damnificados. Los gobiernos local y central tuvieron aciertos y errores en su respuesta a esta calamidad, pues a pesar de recibir apoyo material y moral por parte de otros países, la oposición criticó la decisión de la presidenta de negarse a recibir la ayuda ofrecida por China continental después de conocer que hubo algunos ciudadanos chinos entre las víctimas mortales. No obstante, el gobierno aceptó parte de la asistencia ofrecida por autoridades japonesas, particularmente un equipo de detección de calor, necesario para la localización de sobrevivientes atrapados entre los escombros (Everington, 2018).

Por ser una comunidad altamente dependiente del turismo, el gobierno ofreció medidas para reactivar dicha industria en Hualien durante el mes de marzo, lo que no obstante despertó recelo entre otras zonas de la isla que buscaron atraer mayores incentivos fiscales para el desarrollo de sus propias industrias turísticas (Taipei Times, 16 de marzo). Algunas de esas regiones serían después golpeadas por otros desastres naturales. En el verano de 2018, una serie de lluvias torrenciales azotaron el sur de la isla; grandes porciones de los condados de 
Tainan y Kaohsiung (Gaoxiong) sufrieron inundaciones que duraron varios días. La respuesta gubernamental fue rápida, en especial por tratarse de una región que estaba siendo perjudicada también por una baja en los precios de los productos agrícolas, particularmente frutas y vegetales, debido a la política china de restringir las compras de esos bienes taiwaneses como una de las medidas de presión al gobierno de Tsai. Otras industrias no se vieron tan afectadas, y los programas gubernamentales para promover algunas de ellas se mantuvieron durante 2018.

Ante la demanda de mayores ingresos por parte de los jóvenes, que fue un tópico importante durante las campañas electorales de 2016, el salario mínimo aumentó, aunque no al nivel que muchos esperaban. La economía mostró signos positivos a pesar de las predicciones negativas de principios de año, movida principalmente por una mejora en el consumo interno (China Post, 27 de julio). De igual manera, ante las constantes amenazas de Donald Trump de incrementar la lista de artículos de exportación chinos sujetos a aranceles estadounidenses, las inversiones taiwanesas aprovecharon las políticas del gobierno de Tsai para acercarse a los países del sur y sureste asiáticos, lo que facilitó la diversificación de los destinos de inversiones en el exterior.

El comercio exterior de Taiwán se mantuvo así en números positivos, lo que permitió a la isla seguir rompiendo récords en sus reservas internacionales, al superar los $450 \mathrm{mil}$ millones de dólares a mitad del año, lo que también ha protegido al nuevo dólar taiwanés (NTD) de las presiones cambiarias frente al dólar estadounidense, como las sufridas por las monedas de economías emergentes y de reciente industrialización (China Post, 6 de septiembre). Lo anterior tuvo también un impacto sobre el resto de la economía de la isla, aunque los retos que se observan desde la época de Ma Ying-jeou (Ma Yingjiu) acerca de hacer que las ganancias económicas permearan hasta la base para beneficiar a todos los estratos sociales, y en especial a los jóvenes, siguen lejos de ser resueltos.

\section{Los cambios en el gabinete y el pulso del gobierno con la comunidad universitaria}

A principios de enero, después de un proceso que duró seis meses, el Consejo Universitario de la Universidad Nacional de Taiwán (UNT) elegió a Kuan Chung-Ming (Guan Zhongmin), profesor del Departamento de Finanzas y ex ministro del Consejo de Planeación y Desarrollo Económicos durante el gobierno de Ma (2013-2014), como su nuevo rector. No obstante, el 
Ministerio de Educación fue retrasando el reconocimiento del resultado y eventualmente se negó a ratificar a Kuan en el puesto de rector. Los descontentos con el resultado de la elección comenzaron a interponer denuncias legales contra Kuan, incluyendo acusaciones de plagio y de conflicto de interés por haber participado como asesor de empresas privadas, particularmente la Taiwan Mobile, de la que también fue asesor Richard Tsai (Cai Mingxing), uno de los miembros del comité universitario encargado de supervisar la elegibilidad de los candidatos a rector.

Tres meses después de las elecciones, el 27 de abril, el Ministerio se negó oficialmente a reconocer los resultados e instó a la universidad a realizar nuevos comicios. De igual manera sumó nuevas causas a sus argumentos para evitar que Kuan asumiera el puesto, incluido el hecho de haber dado clases en instituciones de China continental, y posibles violaciones a la Ley de Protección de Información Clasificada sobre Seguridad Nacional (Lin, Wu y Hsiao, 2018). La reacción de las autoridades y de algunos miembros de la comunidad universitaria fue de absoluto rechazo a la posición del gobierno, por lo que declararon que el proceso de selección fue cuidadoso en cada paso, contrarrestando así las acusaciones hechas en contra de Kuan y reclamando el respeto inmediato a la autonomía de la universidad.

Las tensiones entre ambas partes y la intervención de legisladores y otros políticos de alto perfil derivaron en una crisis que forzó la salida del ministro de educación, Pan Wenchung (Pan Wenzhong), en mayo, y la de su sucesor, Wu Maw-kuen (Wu Maokun), en julio. La UNT acusó de parcialidad al gobierno, al haber encontrado que rectores de otras universidades públicas habían sido ratificados por el Ministerio con anterioridad a pesar de existir sospechas de posibles conflictos de interés. En una entrevista radiofónica, la presidenta acusó a Kuan de politizar el asunto, y defendió la posición de su gobierno argumentando que éste estaba comprometido a regirse por las leyes y el Estado de derecho, por lo que debía dejar que las investigaciones siguieran su curso (Shih, 2018).

Luego de una segunda recomposición del gabinete promovida en julio por el primer ministro William Lai (Lai Qingde), el nuevo ministro de educación, Yeh Jiunn-rong (Ye Junrong), se comprometió a resolver el asunto a la brevedad posible. No obstante, para finales de agosto la UNT anunció que no llevaría a cabo nuevas elecciones, y encontró el apoyo de otras grandes universidades públicas, entre las que se encuentran algunas próximas a renovar sus altos mandos, en sus reclamos por la defensa de su autonomía. El ministerio, no obstante, 
emitió finalmente la orden de repetir la última ronda de votaciones, en espera de obtener un resultado más aceptable. Sin embargo, las autoridades universitarias han buscado otros medios en su intento por validar el proceso de elecciones de enero. Indudablemente, si se considera el nivel de influencia de la universidad sobre la política interna de la isla, es posible que la crisis por la que atraviesa haya afectado el resultado de las elecciones locales de noviembre, así como el futuro del gabinete, particularmente en el área de educación.

\section{Reordenamiento político y elecciones locales de noviembre}

Las elecciones de noviembre fueron una prueba para los grandes partidos políticos, en especial para el Kuomintang (Guomindang, KMT), por ser la primera vez que asistía a las urnas sin el gran músculo financiero que utilizó desde su retirada a la isla en 1949. Durante décadas, el entonces partido oficial disfrutó de una red de empresas y propiedades que le permitieron acumular una fortuna considerable que utilizó para propósitos partidistas y electorales, los cuales lo llevaron a convertirse en uno de los partidos políticos más ricos del mundo (Tien, 1989: 85-87). A pesar de haber perdido la presidencia en dos periodos consecutivos, durante la era de Chen Shui-bian (Chen Shuibian) el KMT logró mantener el control del poder legislativo y con ello evitar cambios en sus ingresos. Por dichas razones, cuando el PDP consiguió por vez primera el control del Yuan Legislativo en 2016, una de sus acciones inmediatas fue aprobar una serie de regulaciones para obligar al KMT a separarse de empresas y propiedades bajo su control.

La llamada Ley para el Manejo de las Propiedades Mal Habidas, por parte de partidos políticos y organizaciones afines, de 2016, así como la nueva Ley de Partidos Políticos de 2017, obligaron a todos los partidos políticos a reportar sus propiedades y activos, en particular los obtenidos durante los años de vigencia de la ley marcial. Por medio de dichas leyes, el gobierno de Tsai procedió a congelar gran parte de los activos del KMT, aunque en julio de 2018 se reportó que este partido seguía siendo el más rico de la isla, muy por encima del PDP (Yu y Huang, 2018). Del mismo modo, la elección de Wu Den-yih (Wu Dunyi) como presidente del KMT, un personaje más al centro del espectro político comparado con su antecesora en el puesto, Hung Hsiu-chu (Hong Xiuzhu), no fue suficiente para llevar al partido a los mismos niveles de aceptación que tuvo durante el primer mandato de Ma (20082012). 
Para finales de agosto, una encuesta publicada por un influyente medio de comunicación mostró que por primera vez, desde el triunfo de Tsai Ing-wen, el PDP y el KMT estaban empatados en el nivel de identificación partidista de los encuestados, con $24.5 \%$ cada uno. El mayor grupo de encuestados mostró inconformidad con los dos grandes partidos, y algunos otros se mostraron dispuestos a apoyar a partidos menores o a candidatos independientes (Cui, 2018). Ante ese escenario, las dirigencias de ambos partidos mantuvieron bajas expectativas para las elecciones de noviembre, ya que percibían como un éxito el no perder posiciones y mantener el mismo nivel de electores que tuvieron en los pasados comicios locales de 2014.

Indudablemente las elecciones más observadas serían las de alcaldes en Taipéi (Taibei) y Kaohsiung. Las elecciones de esta última, la mayor ciudad del sur de la isla y un bastión histórico del Partido Democrático Progresista y del movimiento independentista, estuvieron marcadas por la renuncia de Chen $\mathrm{Chu}$ (Chen Ju), la alcaldesa que gobernó la ciudad desde 2006 tras su nombramiento como secretaria general de la Oficina de la Presidencia el mes de abril, y posteriormente como encargada de la campaña del candidato del PDP a la alcaldía de la Ciudad de Nuevo Taipéi (Xinbeishi), Su Tseng-chang (Su Zhenchang). Luego de las crecientes acusaciones por parte de consejeros y políticos locales del KMT sobre la situación de la deuda pública y los crecientes niveles de contaminación en Kaohsiung, así como del natural desgaste de imagen después de tantos años en el puesto, Chen dejó el gobierno de la ciudad en manos del vicealcalde Hsu Li-ming (Xu Liming), quien estuvo a cargo de mantener la alcaldía en manos de PDP, al frente del gobierno de la ciudad desde 1998.

Las elecciones a la alcaldía de Taipéi se caracterizaron por la campaña de reelección de Ko Wen-je (Ke Wenzhe) y por su rompimiento con el PDP, que lo apoyó en su primera elección en 2014. Ampliamente seguido por los jóvenes, Ko fue electo sobre una plataforma en pro de la transparencia y la disciplina fiscal en el gobierno. Por ser candidato independiente, el PDP ofreció su apoyo a Ko en 2014, en una alianza de intereses para impedir el triunfo del KMT y su candidato, Sean Lien (Lian Shengwen), hijo del ex vicepresidente Lien Chan (Lian Zhan). Después de lo que fue percibido como un acercamiento estratégico con el gobierno de China continental, desde su visita a Shanghai en 2017, miembros del PDP pidieron un rompimiento oficial con Ko, y finalmente el partido anunció que no apoyaría al alcalde en su campaña de reelección y que presentaría un candidato propio. 
De esa manera, la elección de 2018 se decidiría entre tres candidatos, aunque la contienda principal sería entre Ko y el candidato del KMT si se considera la fuerte presencia histórica de este último en la capital de la isla. En las elecciones primarias del Kuomintang, el nominado fue el político de corte más conservador, Ting Shou-chung (Ding Shouzhong), quien al conseguir el apoyo de la familia Lien buscó atraer el voto de los sectores más tradicionales y de aquéllos descontentos con el desempeño de Ko. No obstante, el alcalde en funciones se corrió al centro del espectro político en busca de mantener el apoyo no sólo de los jóvenes, sino también de la población inconfome con la política que mostraba la presidenta hacia China continental. Además de promover una agenda de 16 puntos que dan continuidad a la agenda con la que fue electo cuatro años atrás, Ko se reunió con James Soong (Song Chuyu), líder del Partido Primero el Pueblo (pro-unificación), quién manifestó el apoyo oficial del partido a la campaña de reelección de Ko (Chen, 2018).

Luego de esa reunión se incrementaron los rumores acerca de una posible alianza entre los dos políticos para presentar una candidatura conjunta en las elecciones presidenciales de 2020. Ko siguió siendo criticado por sus detractores, especialmente después de negarse a condenar de manera abierta la estrategia de Beijing para quitar aliados diplomáticos a Taiwán luego del rompimiento de vínculos oficiales entre la República de China y El Salvador, y por la aparición de reportajes en la cadena de televisión central de China continental con un tono muy favorable hacia Ko.

Como preparación para las elecciones de noviembre, la administración de Tsai comenzó una campaña oficial en los medios de comunicación de la isla para promover las reformas logradas por el gobierno, las que presentó como un logro importante, especialmente al mostrar imágenes de anteriores presidentes que habían prometido reformas en las mismas áreas y no haberlas cumplido. Algunas leyes en materia de justicia transicional, del ejército y del sistema de pensiones, fueron finalmente aprobadas por el Yuan Legislativo y ratificadas por el Ejecutivo, a pesar de las crecientes protestas por parte de los grupos desfavorecidos ante tales cambios. El descontento de diferentes sectores sociales se manifestó en el creciente grado de desaprobación de Tsai, que por primera vez sobrepasó el 50\%, a la vez que la aprobación bajó hasta $31 \%$, algo que fue interpretado por otros analistas como un signo evidente de la fortaleza del sistema democrático de la isla (Shan, 2018). El resultado de las elecciones de 2018 fue un indicador importante en ese sentido. 


\section{LAS RELACIONES ENTRE AMBOS LADOS DEL ESTRECHO DE TAIWÁN}

Las relaciones entre ambos lados del estrecho se han visto empañadas por diferentes acontecimientos que pusieron en evidencia las dificultades del gobierno de la isla para mantener su espacio internacional, y por continuas acusaciones mutuas de querer cambiar el estatu quo unilateralmente. Durante 2018 el gobierno del continente continuó realizando ejercicios militares en aguas del estrecho, con mayor frecuencia y visibilidad a partir de 2016, así como en los límites del espacio aéreo de la isla. La navegación, el 20 de marzo, del primer portaaviones chino, el Liaoning, por el mar que separa a ambas partes, así como la entrada de aviones militares en la zona de identificación aérea de la isla, fueron percibidas como una señal importante, no sólo para Taiwán sino para Estados Unidos y Japón (Gady, 2018a). Dicho ejercicio evidenció el cambio en el equilibrio de poder de la región, con un claro indicador de que China no estaría dispuesta a permitir una repetición de lo ocurrido más de veinte años atrás, cuando el ejército estadounidense envió parte de la Séptima Flota al estrecho de Taiwán como respuesta al disparo de misiles por parte del Ejército de Liberación Popular a las aguas del estrecho.

De igual manera, las tensiones entre ambos lados crecieron a un ritmo no visto desde la Guerra Fría, cuando el ejército chino llevó a cabo un simulacro de guerra con disparos dentro de su área del estrecho, justo después del mayor desfile de buques y naves de la Marina del ELP en el Mar del Sur de China, observado personalmente por Xi Jinping el 11 de abril. Un día después del desfile, las fuerzas armadas de Taiwán llevaron a cabo su propio simulacro en la costa oriental de la isla, frente al puerto de Su'ao, con Tsai supervisando los ejercicios a bordo de una de las naves. Ésta fue la primera vez que la presidenta observaba personalmente un simulacro, e hizo notar una creciente disposición por parte del ejército y la sociedad para responder a cualquier agresión externa. Aunque ambos ejercicios estuvieron separados por unas horas de diferencia, Tsai hizo notar que el simulacro taiwanés había sido planeado con meses de anticipación, y que se trató de una mera coincidencia (Hamacher, 2018). No obstante, quedó claro que con el gobierno de Tsai ambos lados del estrecho han tomado más en serio las posibilidades de recurrir a las armas para detener cualquier intento, unilateral y violento, por cambiar la situación actual hacia las opciones de reunificación o separación definitivas. 
Por dichas razones, el gobierno de la isla anunció también un incremento considerable en el presupuesto de defensa para los próximos años, en el que destaca la promoción de un programa destinado a mejorar las defensas aérea y naval de la isla, particularmente en lo relacionado con la construcción de una flota de submarinos propios (Gady, 2018b). Como veremos más adelante, dicho programa ha sido bien recibido por algunos de los tomadores de decisiones en Washington, quienes están dispuestos a otorgar algunas de las partes necesarias para la fabricación de dichas naves. Ante la creciente imposibilidad de recurrir al mercado mundial de armas, con una creciente presión por parte de Beijing a otros países para no proveer de armamento a la isla, es probable que, de concretarse, la flota de submarinos taiwanesa se pueda hacer únicamente con asistencia estadounidense y, en menor medida, japonesa, y sólo después de haber sorteado una serie de obstáculos planteados por el gobierno continental.

La negativa del gobierno de Tsai por reconocer el Consenso de 1992 como la base de las relaciones entre ambos lados del estrecho ha llevado al gobierno de Xi a incrementar sus mecanismos de presión para reducir aún más el espacio internacional de la isla. Dichos mecanismos han sobrepasado el tradicional ámbito diplomático entre gobiernos y se ha trasladado al campo de los negocios. Desde 2017, el gobierno chino comenzó a presionar a grandes compañías multinacionales, principalmente en el sector de los servicios, para cambiar la nomenclatura en sus sitios web. Cualquier referencia que pudiera llevar a pensar en Taiwán como un país independiente o separado de China sería respondida por un duro llamado a boicotear la marca por parte de los internautas chinos.

Una serie de compañías comenzó entonces a cambiar sus referencias, de "Taiwán" a “Taiwán, provincia de China”, o simplemente "Taiwán, China”. El uso anterior de nombres más ambiguos, como "Taipéi Chino" o "China Taipéi”, fue menos aceptado esta vez. El haber cedido a las presiones para evitar perder la cuota en un mercado de más de mil millones de potenciales consumidores fue respondido con duras críticas por parte del gobierno y la sociedad de la isla. No obstante, incapaces de competir con el mercado chino, el Ministerio de Asuntos Exteriores de Taiwán se limitó a enviar notas de insatisfacción a dichas compañías y a hacer un llamado a volver a la numenclatura anterior (Chuan y Hsu, 2018).

En 2018 la presión se dio en las compañías aéreas internacionales, con la gran mayoría de ellas permitiendo eventualmente cambiar el nombre en sus sitios web. Las aerolíneas 
estadounidenses crearon un frente común para resistir a las presiones, buscando el apoyo de su gobierno para mediar ante lo que fue visto como una interferencia por parte de un gobierno extranjero. A pesar de que funcionarios del Departamento de Estado estadounidense condenaron la presión del gobierno chino, incluida una amenaza de imponer sanciones económicas a las compañías que se resistieran a cambiar el nombre, justificándose en una violación de la soberanía china, las empresas procedieron a modificar la referencia utilizada para la isla. Luego de que Beijing pusiera una fecha límite para hacer el cambio, y con los funcionarios del gobierno inmersos en negociaciones relacionadas con la imposición unilateral de aranceles, las grandes aerolíneas estadounidenses fueron de las últimas en el mundo en llevar a cabo la modificación (Paquette, 2018). No obstante, al contrario de lo ocurrido con otras compañías, las firmas aéreas de Estados Unidos se limitaron a utilizar únicamente el nombre de las ciudades de la isla, y dejaron de usar Taiwán para referirse simplemente a Taipéi, Taichung (Taizhong), Kaohsiung, etcétera.

Otro motivo de tensión que tuvo un impacto notable en la sociedad taiwanesa ocurrió a mediados de 2018, luego de que el 24 de julio, en una sesión extraordinaria del Comité Olímipico del Este Asiático (EAOC, por sus siglas en inglés) convocada por representantes chinos, se votó a favor de la cancelación de los derechos de Taichung para ser la sede de los primeros Juegos de la Juventud del Este Asiático. ${ }^{3}$ Los juegos, originalmente planeados para ser llevados a cabo en 2019, habían sido otorgados a la ciudad taiwanesa en 2014. No obstante, desde principios de 2018 una serie de organizaciones civiles en la isla comenzó una campaña de recolección de firmas para pedir la realización de un referendo sobre un cambio de nombre en el equipo olímpico. El movimiento busca que el equipo representativo de la isla participe en los juegos olímpicos de Tokio 2020, no con el nombre que ha usado desde su regreso a las justas olímpicas, "China Taipéi”, sino simplemente como Taiwán. El gobierno chino se opuso desde el principio a dicha campaña, y la cancelación de los juegos en Taichung fue vista como el último intento por influir sobre el resultado de ésta.

${ }^{3}$ El Comité Olímpico del Este Asiático es el organismo subregional encargado de promover el deporte y los eventos olímpicos en esa parte del mundo. Está conformado por nueve delegaciones que representan los comités olímpicos locales (China, Corea del Norte, Corea del Sur, Guam, Hong Kong, Japón, Macao, Mongolia y China Taipéi). De los nueve representantes presentes en la sesión extraordinaria donde se decidió cancelar los derechos de Taichung, sólo el representante taiwanés votó en contra y el representante japonés se abstuvo; el resto de representantes votó a favor de la moción propuesta por el representante de China continental (Hsu, 2018). 
No obstante, la noticia de la revocación de los derechos de la ciudad para albergar los juegos, a menos de un año de su realización, fue muy mal recibida ante la sociedad de la isla, incluso ante la población escéptica o desinteresada en participar en el referendo. Así, semanas después del anuncio se registró un incremento en la recolección de firmas para la campaña, hasta sobrepasar el mínimo requerido por la legislación para convocar a dicha consulta, y el gobierno local de Taichung anunció una impugnación ante el Comité Olímpico Internacional, así como su disposición para proceder con la justa deportiva, aunque tuviera que hacerlo con otro título, el de los primeros Juegos de la Juventud de Asia-Pacífico. De igual modo, la respuesta inmediata del alcalde de esa ciudad, Lin Chia-lung (Lin Jialong), del PDP, elevó su perfil nacional y le colocó como favorito para ganar la reelección en los comicios de noviembre, a pesar de que la ciudad ha sido históricamente un lugar de apoyo del KMT.

Por otra parte, a pesar del uso cada vez más frecuente de medidas punitivas y de presión, el gobierno chino no dejó de recurrir a incentivos para los habitantes y empresarios de la isla, así como a hechos encaminados a distender un poco las relaciones entre ambos lados del estrecho. Como una forma de ganar los corazones, y los bolsillos, de los taiwaneses, en marzo de 2018 la Oficina para Asuntos de Taiwán del Consejo de Estado de la RPCh anunció 31 medidas encaminadas a atraer mayores inversiones de empresas taiwanesas, así como capital humano proveniente de la isla. Las medidas, implementadas por decenas de ministerios y oficinas del gobierno central, consisten en el otorgamiento de facilidades para el establecimiento de firmas y personas procedentes de Taiwán en China continental, en las áreas de comercio, industria, finanzas, educación, ciencia y tecnología, entre muchas otras (19 medidas destinadas a los sectores sociales y de empleo, y 12 para el sector de negocios).

Con la economía china creciendo todavía por encima del rango de $6 \%$ anual, es natural que cientos de taiwaneses, incluidos los más jóvenes, se sintieron atraídos a migrar a China continental en búsqueda de mejores oportunidades (Denyer, 2018). Conscientes de esta tendencia, las autoridades continentales han trabajado para seguir atrayendo el talento de la isla, así como convenciendo a empresarios locales de seguir moviendo parte de su producción a China continental, ofreciéndoles un trato preferencial, similar al recibido por los fabricantes locales chinos. Críticos y algunos analistas aseguran que las medidas podrían ir en contra de las reglas de la OMC, por su naturaleza discriminatoria, y que su efectiva implementación y coordinación puede tardar mucho tiempo, por el entramado burocrático imperante en China (Bush et al., 2018: 10). 
Además, dadas las condiciones de incertidumbre derivadas de los anuncios de imposición de aranceles a los productos chinos por parte de Estados Unidos, y lo que se percibe como un creciente apoyo de los gobiernos provinciales y locales a los fabricantes domésticos, las empresas taiwanesas han dejado de migrar en masa hacia el continente y han empezado a deslocalizar su producción hacia otras partes de Asia y el mundo emergente. Las medidas anunciadas pueden servir entonces para detener, aunque no se sabe si para revertir, el flujo de capitales procedentes de la isla a China continental. De cualquier manera, ante el anuncio de las 31 medidas, el gobierno en Taiwán respondió con una serie de políticas propias, con menos claridad en su consistencia, encaminadas a evitar una fuga de cerebros y de capital hacia el otro lado del estrecho.

Otro paso para demostrar la voluntad del gobierno de la RPCh en su afán por reducir las tensiones entre ambos grupos fue anunciado el 13 de julio, cuando Lien Chan, anterior presidente del KMT y ex vicepresidente de la República de China en Taiwán, viajó a Beijing acompañado de una pequeña comitiva para encontrarse con Xi Jinping. La reunión se vivió en un clima de cordialidad, con ambos políticos reconociendo la necesidad de desarrollar relaciones pacíficas entre los dos lados del estrecho, y llamando a Tsai y a su gobierno a aceptar el Consenso de 1992 como la base de dicho desarrollo (Xinhua, 13 de julio). A pesar de que Hung Hsiu-chu había viajado a China continental el año anterior, en su calidad de presidenta del KMT, ésta era la primera vez que Xi Jinping se reunía personalmente con un político de alto perfil de la isla desde su encuentro con Ma Ying-jeou en Singapur. Para el KMT, la reunión con Xi fue vista como una oportunidad para ganar adeptos entre los inconformes con la política de Tsai hacia el continente, en la antesala de las elecciones locales de 2018. La reunión sirvió también para que el gobierno chino reforzara la importancia de las 31 medidas, en un intento por consolidar su estrategia de palos y zanahorias en su trato hacia el gobierno de Tsai y hacia los habitantes de la isla en general.

De esa manera, a pesar de los incentivos económicos, la creciente presión sobre los actores internacionales públicos y privados responde no sólo a limitar el espacio de la isla ante lo que se percibe como un intento de Tsai por cambiar el statu quo y llevarla a aceptar el Consenso de 1992, sino que también al empeoramiento de las relaciones entre los gobiernos de China y Estados Unidos. Frente a las medidas encaminadas hacia una guerra comercial desatada por Donald Trump, así como otros puntos de presión buscados por 
Washington para forzar a Beijing a ofrecer mayores concesiones económicas a la parte estadounidense, el gobierno de Xi ha respondido con una mayor presión sobre la isla.

Igualmente, luego de que la Constitución de la RPCh fue reformada para permitir la reelección de Xi después de su segundo mandato, la presión sobre este último para resolver la cuestión de Taiwán durante su administración se ha incrementado. Con más años al frente del gobierno, se espera que el presidente chino pueda lograr la reunificación del territorio, algo que ha sido un reto constante de los diferentes líderes comunistas a lo largo del tiempo, especialmente entre los llamados "halcones" en el aparato militar y otros grupos dentro del Partido Comunista Chino (Bush et al., 2018: 25). Por ello, ante las dificultades que se han presentado en el frente exterior en los últimos años, y la falta de apertura política en el sistema, la resolución de problemas como el de Taiwán se ha vuelto urgente para una parte importante del grupo dirigente de la RPCh. Sin embargo, ante dicha situación, el gobierno de la isla ha buscado resistir a las presiones probando nuevas formas no sólo de evitar el creciente aislamiento y limitación de su espacio internacional, sino incluso de revertirlo, aunque sea con claroscuros en el camino.

\section{LAS RELACIONES EXTERIORES DE LA ISLA}

Durante uno de los debates efectuados rumbo a las elecciones presidenciales en las que resultó electa, Tsai denunció que la supuesta tregua diplomática del gobierno de Ma Yingjeou con Beijing, en la que ambos lados evitaron disputarse países aliados, había tenido el efecto negativo de hacer perder el rumbo a los diplomáticos de la República de China (Hsu, 2015). Por lo tanto, ella propuso reencauzar los esfuerzos de los funcionarios del servicio exterior para así lograr aumentar la presencia de Taiwán en el escenario internacional. Una vez en el poder, Tsai y su gobierno anunciaron la búsqueda de un mayor contacto con los aliados no oficiales más poderosos, especialmente Estados Unidos, Japón y la Unión Europea, así como un proyecto para acercarse a los países de sureste y sur de Asia. Dicha estrategia en política exterior ha presentado resultados positivos y negativos, ya evidenciados a lo largo de 2018.

La nueva retórica del gobierno de Trump frente a China también ha tenido un impacto en las relaciones de Estados Unidos con Taiwán. La necesidad de encontrar puntos de presión sobre el gobierno chino para lograr mayores concesiones comerciales ha coincidido con un 
mayor acercamiento del gobierno estadounidense hacia las autoridades de la isla. Desde principios de 2018, la ratificación de la Ley de Viajes a Taiwán, que incluye mayores intercambios y visitas entre funcionarios de alto nivel de los dos países así como la inclusión de artículos referentes a la defensa de Taiwán en la Ley de Autorización de Defensa Nacional, han sido percibidas como evidencia del apoyo de Washington a Taipéi, y como una señal a Beijing para evitar la modificación unilateral del statu quo.

En ese sentido, la ley antes mencionada destina 716 mil millones de dólares al presupuesto de defensa estadounidense, con una partida para incrementar la cooperación con las fuerzas armadas de la isla, no sólo en cuanto al mejoramiento de sus capacidades de defensa, sino también para el intercambio de conocimiento en materia humanitaria y de asistencia en desastres naturales (Lu y Chung, 2018). A pesar de enfatizar esta última parte para evitar dar la impresión de haberse desviado de su política de Una Sola China, el Departamento de Estado también procedió a aprobar el otorgamiento de licencias a empresas estadounidenses interesadas en dotar de tecnología, partes y equipo a empresas taiwanesas involucradas en el proyecto para la construcción de una flota doméstica de submarinos. De esa manera, Estados Unidos se erigió como el único país con la capacidad y voluntad para apoyar a las autoridades taiwanesas en su plan de iniciar en 2020 la fabricación de un submarino nuclear propio, con la idea de concluirlo en 2024. Lo anterior también ha sido visto con cautela por algunos expertos estadounidenses en seguridad, quienes han cuestionado la dificultad en la posible identificación de los submarinos taiwaneses frente a los submarinos chinos, en un escenario de conflicto internacional en aguas del estrecho de Taiwán (Gady, 2018b).

En otra muestra de continuo apoyo a la isla, el gobierno estadounidense inauguró la nueva sede del American Institute in Taiwan (AIT), que funge como una embajada no oficial en la isla. Construida sobre un terreno más amplio y con un mayor espacio para oficinas, incluidas la de representantes del ejército estadounidense, la nueva sede se convirtió también en un asunto delicado dentro de las relaciones bilaterales entre Washington y Beijing. Programada para el mismo día del encuentro entre Donald Trump y Kim Jong-un en Singapur, el 12 de junio la inauguración del AIT en Taipéi supuso un reto para el gobierno estadounidense en lo relacionado con la elección del representante gubernamental que presidiría el evento (Bush, 2018). 
A pesar de que la ratificación de la nueva Ley de Viajes permite la visita de altos funcionarios del ejecutivo estadounidense a Taiwán, en la práctica Washington se ha enfrentado a una negativa por parte de Beijing para otorgar visados a aquellos funcionarios estadounidenses que hayan visitado la isla en carácter oficial. Por ello, la ceremonia de inauguración contó finalmente con la presencia de Mary Rogers, subsecretaria de Estado para Asuntos de Educación y Cultura, así como del legislador Gregg Harper y los miembros del cuerpo diplomático encargados del funcionamiento del instituto. Aunque en Taiwán esto fue visto como un signo positivo del compromiso estadounidense hacia la isla, quedó claro que el gobierno norteamericano conservaría su característica ambigüedad estratégica frente a ambos lados del estrecho de Taiwán.

No obstante, en aras de mostrarse como un garante de la paz y de estabilidad regionales, luego de las crecientes tensiones entre ambos lados del estrecho con los ejércitos del continente y de la isla llevando a cabo simulacros militares a gran escala, el 9 de julio dos buques de guerra estadounidenses de la Flota del Pacífico entraron en aguas del estrecho de Taiwán, en lo que fue anunciado como un patrullaje de rutina (Kim, 2018). Igualmente, a finales de septiembre se dieron a conocer los planes estadounidenses de vender partes de armamento y aviones de combate a las autoridades de la isla, por un costo mayor a los 330 millones de dólares, en lo que sería considerada como la segunda venta de armas a Taiwán durante la administración de Trump. No obstante, ésta se llevaría a cabo a una escala mucho menor que la que tuvo lugar en 2017. Dichas maniobras se enmarcan no sólo en las relaciones triangulares entre Estados Unidos, Taiwán y China continental, sino que también han sido percibidas por Beijing como otro elemento de presión en los conflictos comerciales bilaterales sostenidos con Washington.

Por otra parte, el gobierno de la isla buscó presentar como exitosas las escalas hechas por la presidenta Tsai en Estados Unidos durante su gira en agosto por América Latina. Durante su viaje de ida a Paraguay, donde asistió a la toma de posesión del nuevo presidente Mario Abdo Benítez, Tsai hizo una escala en Los Ángeles, donde fue recibida por miembros de la comunidad taiwanesa en California, así como por legisladores y otros funcionarios estadounidenses afines a Taiwán. Después de Asunción, Tsai pasó unos días en Belmopán, donde se reunió con el primer ministro beliceño, Dean Barrow, antes de retornar a Taiwán. En su viaje de regreso, la presidenta volvió a hacer escala en Estados Unidos, esta vez en Houston. Ambas escalas se distinguieron de ocasiones anteriores en las que líderes de la isla 
visitaron ciudades estadounidenses. Ahora, por vez primera, Tsai pudo visitar las sedes de oficinas representativas de Taiwán en ciudades norteamericanas, las cuales otorgan una mayor visibilidad a la representación del gobierno de la isla en el exterior. La presidenta también fue recibida en edificios públicos estadounidenses, como la Biblioteca y Museo Presidencial de Ronald Reagan en California, donde ofreció un discurso ante los medios, y el Centro Espacial Lyndon B. Jhonson de la NASA, en Texas (Chung, 2018a). De esa manera, las autoridades de Taiwán presentaron las escalas de Tsai como un éxito en las estrategias de acercamiento con Estados Unidos, y de mayor visibilidad de la isla en el escenario internacional.

Sin embargo, la respuesta de Beijing no se hizo esperar, y horas después del regreso de la presidenta a la isla, el Ministerio de Relaciones Exteriores de la República de China en Taiwán tuvo que anunciar el rompimiento de relaciones diplomáticas con El Salvador, adelantándose así al anuncio oficial del establecimiento de vínculos oficiales entre este último y la RPCh. Así el gobierno de Taiwán se quedó con 17 aliados, con el salvadoreño como el tercer gobierno en romper relaciones con la isla en 2018. Luego de empezar el año con 20 aliados, entre abril y mayo, en menos de un mes los gobiernos de República Dominicana y Burkina Faso anunciaron el establecimiento de relaciones diplomáticas con su contraparte de la República Popular China.

El gobierno en la isla acusó a Beijing de buscar modificar el estatu quo de manera unilateral, reviviendo la estrategia conocida como diplomacia del dólar, característica de la Guerra Fría y de las primeras décadas posteriores a ésta. El rompimiento de relaciones con el gobierno de Burkina Faso dejó a Taiwán con un solo aliado diplomático africano, lo que coincidió con la visita de Tsai a Suazilandia, conocida ahora con el nombre de eSwatini, para celebrar el quincuagésimo aniversario de las relaciones entre ambos países. La visita fue calificada como un esfuerzo taiwanés por preservar a su único interlocutor oficial en África. No obstante, los medios de comunicación internacionales comenzaron a cuestionar la duración de dicha alianza, especialmente de cara a la Cumbre China-África, mencionando la creciente presión de Beijing sobre el gobierno del pequeño reino para romper relaciones con la isla (Chung, 2018b); estos rumores fueron negados continuamente por ambas partes. Sin embargo, dichos medios también comenzaron a reconocer la creciente presión china sobre los diferentes gobiernos de países africanos, para ahuyentar las inversiones de empresas taiwanesas como una manera de aislar al gobierno y firmas de la isla en esa parte del mundo. 
Por otro lado, el rompimiento con el gobierno de El Salvador causó una reacción inesperada, no sólo en Taiwán, sino también en Estados Unidos. Con cierta diferencia respecto de rompimientos anteriores durante el gobierno de Tsai, en el anuncio oficial del Ministerio de Relaciones Exteriores de la isla éste acusó a su contraparte salvadoreña de haber elegido a Beijing luego de que Taipéi rechazara sus peticiones de otorgar financiamiento para completar la construcción de un puerto en las costas del Pacífico, el Puerto La Unión, que sería también de utilidad para el partido oficial de cara a las próximas elecciones en el país centroamericano (Gu y Chen, 2018). San Salvador negó dichas acusaciones ante duras críticas de partidos y políticos de oposición, aunque el gobierno presentó su decisión desde la lógica de contribuir al desarrollo económico del país mediante el incremento de los flujos comerciales y de inversión con China continental.

De igual modo, el gobierno estadounidense mostró su preocupación específicamente por las implicaciones de inversiones chinas en la construcción de infraestructura estratégica en Centroamérica. Luego de los rompimientos de Taiwán con Panamá, República Dominicana y El Salvador, legisladores estadounidenses, incluido el senador republicano Marco Rubio, presentaron una propuesta de ley para concretar mecanismos de presión y de atracción para impedir que la isla continúe perdiendo aliados diplomáticos, particularmente los ubicados en la región de América Latina y el Caribe. Algunos políticos estadounidenses también sugirieron que el gobierno estadounidense podría revisar o incluso congelar la asistencia otorgada a El Salvador, con el fin de hacer llegar un mensaje contundente acerca de las consecuencias de recibir ayuda china en esa región. El Departamento de Estado anunció también la llamada a consultas de sus embajadores en dichos países latinoamericanos, para conocer más a detalle las razones detrás del establecimiento de vínculos oficiales con Beijing (CNA, 13 de septiembre). Ante tal reacción por parte de Washington, es posible que el éxodo de países centroamericanos se detenga temporalmente, a pesar de las voces que han sugerido que después de El Salvador era posible que los otros dos países que conforman el llamado Triángulo del Norte centroamericano, Guatemala y Honduras, siguieran inmediatamente a su vecino en el establecimiento de relaciones diplomáticas con la República Popular China.

El rompimiento de relaciones con El Salvador fue aprovechado también por la oposición en la isla para criticar la política exterior implementada por Tsai. Incluso Ma Yingjeou utilizó dicho acontecimiento para confirmar la existencia de una tregua diplomática con 
Beijing durante su gobierno (Lee y Chang, 2018). El KMT y otros políticos hicieron un llamado para esforzarse en detener la pérdida de aliados, a lo que el Ministerio de Relaciones Exteriores respondió que ya trabaja en un plan que incorpora todas las posibilidades, incluido el escenario remoto de que la isla se quede sin ningún país con el que pueda mantener relaciones diplomáticas.

Por otro lado, en su propio vecindario el gobierno de la isla trabajó por seguir mejorando sus relaciones con otros países. La relación con Japón se mantuvo en buenos términos, aunque hubo algunos episodios que provocaron tensiones bilaterales y algunos problemas al interior de la isla. La instalación de una estatua en Tainan para conmemorar las víctimas del comercio sexual durante los años de la ocupación japonesa, conocidas en inglés como "comfort women", particularmente en la Segunda Guerra Mundial, produjo molestia entre viajeros y políticos nipones en la isla. Asimismo, la publicación de declaraciones no oficiales hechas a medios japoneses por el expresidente Chen Shui-bian desde su prisión domiciliaria, provocó molestia entre algunos políticos y medios de Taiwán (Hetherington, 2018). Otra dificultad al interior del Ministerio de Relaciones Exteriores de la isla se suscitó a mediados de septiembre, después del suicidio del titular de la Oficina Representativa de Taiwán en Osaka, luego de la presión recibida por parte del gobierno y de las redes sociales debido a los problemas derivados del impacto de un super tifón sobre los miles de turistas taiwaneses en aquella ciudad, incluida la cancelación de decenas de vuelos que obligaron a ciudadanos de la isla a permanecer varios días en Japón. Por otra parte, el gobierno de la isla selló varios acuerdos en materia turística con su contraparte de Corea del Sur, lo que aumentó el flujo de turistas entre ambos países (Chiu y Shih, 2018).

Durante 2018 el gobierno continuó desarrollando la llamada Nueva Política Hacia el Sur, con lo que buscó incrementar sus contactos con los países del sureste y sur de Asia, además de con Australia y Nueva Zelanda. La política ha ofrecido resultados mixtos, aunque parece existir una implementación más sistemática que los intentos anteriores de los gobiernos de Lee Teng-hui y Chen Shui-bian por acercarse a esas regiones (Glaser et al., 2018). En ese sentido, los gobiernos de países como Filipinas e Indonesia se mostraron abiertos a discutir ciertos temas con su contraparte en Taiwán, particularmente en lo relacionado con la situación de los trabajadores migrantes de esos países en la isla. El gobierno en Taiwán también se mostró más flexible en su política de levantar las restricciones 
de visados para los turistas provenientes de esas regiones, aún sin demandar reciprocidad por parte de los otros países.

De igual manera, el gobierno anunció que aunque el presupuesto de 2019 para el establecimiento de la nueva política sería menor que el del año anterior, en la práctica la suma destinada sería mayor. Es decir, el nuevo presupuesto de la Nueva Política Hacia el Sur no incluye la ayuda a la cooperación para el desarrollo de esos países, que se anexó en otro rubro, por lo que los fondos disponibles para la ejecución de esa política aumentarían en 2019 (Lee, Yang y Chin, 2018). A pesar de ello, es poco probable que dichas medidas tengan un impacto inmediato en las relaciones de Taiwán con esas naciones, particularmente frente a las crecientes presiones por parte del gobierno de China continental para seguir aislando a las autoridades de la isla en el escenario internacional. Ello se vio reflejado también en la falta de invitación, por segundo año consecutivo, para la asistencia de Taiwán en calidad de observador ante la Asamblea de la OMS y la Organización Internacional de Aviación Civil, foros en los que había participado durante los años de gobierno de Ma. Ha sido por esos motivos que los cambios en la política exterior de Tsai respecto a su antecesor, en busca de un incremento en la visibilidad y espacio internacionales de la isla, han arrojado resultados mixtos a lo largo de 2018.

\section{CONSIDERACIONES FINALES}

Las crecientes tensiones entre los gobiernos de Estados Unidos y la RPCh, así como entre los dos lados del estrecho de Taiwán, han puesto a la isla en una posición complicada y nuevamente en el centro de atención de los estudiosos de las relaciones internacionales especializados en la región Asia-Pacífico. Desde el cambio de gobierno en 2016, la presidenta ha buscado promover una serie de reformas presentadas como la solución a los retos económicos y sociales que ha enfrentado la isla en las primeras décadas de este siglo. Las elecciones locales de 2018 fueron vistas como un referendo a dichas políticas y a la postura que ha tenido el gobierno hacia su contraparte en China continental.

Sin embargo los resultados han mostrado también que los taiwaneses mantienen sus convicciones democráticas, lo que sigue alejando a los jóvenes de la creencia de que ambos lados del estrecho pertenecen a una misma familia. Ello pone más presión sobre los tomadores de decisiones en Beijing para encontrar una forma de acelerar la reunificación sin 
desatar una reacción negativa por parte de los países vecinos y otras potencias mundiales, particularmente de Estados Unidos, cuyo gobierno está compometido legalmente a defender a la isla en caso de un intento unilateral por modificar su statu quo.

A pesar de un complicado escenario en 2018, la isla ha mostrado su resiliencia en muchos frentes, aunque en los siguientes meses probarán ser definitivos de cara a las elecciones presidenciales de 2020. En dichos comicios Tsai buscará, primero, ser nominada por su partido para buscar la reelección, y después, conseguir la victoria sobre los candidatos del KMT y otros partidos. De mantenerse el nivel de presión por parte de Beijing, y luego de los resultados de las elecciones intermedias en Estados Unidos, la situación de Taiwán para los próximos meses parece traer más dilemas que soluciones.

\section{REFERENCIAS BIBLIOGRÁFICAS}

Bush, R. (2018), "Why a New Office Building in Taiwan is Heightening U.S.-China Tensions", Brookings: Order from Chaos, 29 de mayo. Recuperado de: https://www.brookings.edu/blog/order-from-chaos/2018/05/29/why-a-new-officebuilding-in-taiwan-is-heightening-u-s-china-tensions/

Bush, R., B. Glaser, S. Rigger, R. Hass y R. Wang (2018), Taiwan Under Tsai: A Two-Year Review, foro académico realizado el 2 de mayo, Washington D.C., The Brookings Institution.

Chen, J. (2018), “Ke Wenzhe, Song Chuyu Zai Tongtai-Juzi Fu Baishuang (Ko Wen-je y James Soong en la misma plataforma-los naranjas se cubren de escarcha), Lianhebao (United Daily News), 27 de agosto. Recuperado de: https://udn.com/news/story/11311/3332042

China Post (2018), “Academia Sinica Rises Taiwan's 2018 GDP Growth Forecast”, China Post, 27 de julio. Recuperado de: https://chinapost.nownews.com/20180727-387005

China Post (2018), “Taiwan's Foreign Exchange Reserves Hit Record High in August", China Post, 6 de septiembre. Recuperado de: https://chinapost.nownews.com/20180906-414018 
Chiu, C. y H. Shih (2018), "Taiwan, South Korea Launch Reciprocal E-Gate Clearance Program", Focus Taiwan, 27 de junio. Recuperado de: http://focustaiwan.tw/news/asoc/201806270030.aspx

Chuan, K. y E. Hsu (2018), "Taiwan Condemns Beijing's Request to Airlines for Jeopardizing Universal Values", China Post, 29 de mayo. Recuperado de: https://chinapost.nownews.com/20180529-334841

Chung, L. (2018a), “United States 'Plays Taiwan Card' With Tsai Ing-wen's Trip to NASA's Mission Control Center", Político, 20 de agosto. Recuperado de: https://www.politico.com/story/2018/08/20/tsai-ing-wen-taiwan-president-nasa-visit788931

Chung, L. (2018b), "Taiwanese Businesses Feel the Squeeze in Africa as Beijing Extends its Reach", South China Morning Post, 24 de agosto. Recuperado de: https://www.scmp.com/news/china/diplomacy-defence/article/2161262/taiwanesebusinesses-feel-squeeze-africa-beijing

CNA (2018), "State Department Summons Envoys in Support of Taiwan”, Taipei Times, 13 de septiembre. Recuperado de: http://www.taipeitimes.com/News/taiwan/archives/2018/09/13/2003700331

Cui, C. (2018), "Minjindang Rentong de Shishang Zuidi-Lan Lü Shili Jiejin (El nivel de Reconocimiento del PDP es el más bajo de la historia-El poder de azules y verdes empatado)", Zhongguo shibao (China Times), 20 de agosto. Recuperado de: https://www.chinatimes.com/realtimenews/20180820001305-260407

Denyer, S. (2018), “Taiwan Battles a Brain Drain as China Aims to Woo Young Talent”, The Washington Post, 15 de abril. Recuperado de: https://www.washingtonpost.com/world/asia_pacific/taiwan-battles-a-brain-drain-aschina-aims-to-woo-young-talent-away/2018/04/13/338d096e-3940-11e8-af3c$2123715 \mathrm{f} 78 \mathrm{df} \_$story.html?utm_term $=.12973 \mathrm{~cd} 2 \mathrm{e} 7 \mathrm{ea}$

Everington, K. (2018), “Taiwan Rejects Offer of Help from China, Accepts Japanese Expertise", Taiwan News, 9 de febrero. Recuperado de: https://www.taiwannews.com.tw/en/news/3360308 
Gady, F. (2018a), "China Sends Aircraft Carrier Through Taiwan Strait”, The Diplomat, 22 de marzo. Recuperado de: https://thediplomat.com/2018/03/china-sends-aircraftcarrier-through-taiwan-strait/

Gady, F. (2018b), “Taiwan's Indigenous Submarine to Be Based on European Design”, The Diplomat, 26 de septiembre. Recuperado de: https://thediplomat.com/2018/09/taiwans-indigenous-submarine-force-to-be-basedon-european-design/

Glaser, B., S. Kennedy, D. Mitchell y M. Funaiole (2018), The New Southbound Policy: Deepening's Taiwan Regional Integration. A Report of the CSIS China Power Project", Washington D.C., Center for Strategic and International Studies/Rowman \& Littlefield

Gu, Q. y J. Chen (2018), Meifang ceng shijing lianhegang wenti xuezhe: Sa'erwaduo guzhuyizhi (Estados Unidos había advertido del problema del Puerto La Unión. Experto dice: El Salvador puso todos sus huevos en una canasta)", Zhongyang tongxun she (Central News Agency), 21 de agosto. Recuperado de: http://www.cna.com.tw/news/aipl/201808210129.aspx

Hamacher, F. (2018), “Taiwan President Joins Naval Drill a Day After Huge China Exercise”, Reuters, 13 de abril. Recuperado de: https://www.reuters.com/article/us-taiwanmilitary-drills/taiwan-president-joins-naval-drill-a-day-after-huge-china-exerciseidUSKBN1HK0RC

Hetherington, W. (2018), "Ex-President Urges Independence Poll”, Taipei Times, 6 de septiembre. Recuperado de: http://www.taipeitimes.com/News/taiwan/archives/2018/09/06/2003699892

Hsu, S. (2018), "Taichung Loses Right to Host Games", Taipei Times, 25 de julio. Recuperado de: http://www.taipeitimes.com/News/front/archives/2018/07/25/2003697320

Hsu, S. (2015), "Ma Blasts Tsai over 'Diplomatic Truce”, Taipei Times, 30 de diciembre. Recuperado de: http://www.taipeitimes.com/News/taiwan/archives/2015/12/30/2003636013 
Kim, J. (2018), "US Navy Warships Sail Through Taiwan Strait Amid China Tensions", China Post, 9 de julio. Recuperado de: https://chinapost.nownews.com/20180709372957

Lee, S. y S. C. Chang (2018), "Ex-President Ma: Do Something to Stop Losing More Allies”, China Post, 22 de agosto. Recuperado de: https://chinapost.nownews.com/20180823404555

Lee, H., M. Yang y J. Chin (2018), "New Southbound Policy Budget to be Boosted”, Taipei Times, 21 de agosto. Recuperado de: http://www.taipeitimes.com/News/taiwan/archives/2018/08/21/2003698906

Lin, R., P. H. Wu y S. Hsiao (2018), "Ministry Rejects Appointment of NTU President-Elect", Taipei Times, 28 de abril. Recuperado de: http://www.taipeitimes.com/News/front/archives/2018/04/28/2003692128

Lu, Y. y J. Chung (2018), “Taiwan Grateful to US for Defense Act”, Taipei Times, 15 de agosto. Recuperado de: http://www.taipeitimes.com/News/front/archives/2018/08/15/2003698543

Paquette, D. (2018), "Under Pressure from China, U.S. Airlines Start Changing References to Taiwan", Washington Post, 25 de julio. Recuperado de: https://www.washingtonpost.com/world/under-pressure-from-china-americanairlines-changes-references-to-taiwan-on-its-website/2018/07/25/1b302984-8fc911e8-9b0d-749fb254bc3d_story.html?noredirect=on\&utm_term=.b04897eb50e5

Shan, S. (2018), "Tsai's Approval Rating Drops to 31.2\%", Taipei Times, 18 de septiembre. Recuperado de: http://www.taipeitimes.com/News/taiwan/archives/2018/09/18/2003700647

Shih, H. (2018), "NTU's Kuan 'Politicizing' Case: President Tsai”, China Post, 14 de mayo. Recuperado de: https://chinapost.nownews.com/20180514-317961

Taipei Times (2018), “Tourism Bureau to Offer Hualien Travel Subsidies to Couples”, Taipei Times, 16 de marzo. Recuperado de: http://www.taipeitimes.com/News/taiwan/archives/2018/03/16/2003689404 
Tien, H. M. (1989), The Great Transition. Political and Social Change in the Republic of China, Taipei, SMC Publishing

Xinhua (2018), "Xi Jinping Meets with Taiwan Delegation Led by Lien Chan", Global Times, 13 de julio. Recuperado de: http://www.globaltimes.cn/content/1110672.shtml

Yu, K. H. y F. Huang (2018), “KMT Owns Largest Assets of All Political Parties in Taiwan”, Focus Taiwan, 13 de julio. Recuperado de: http://focustaiwan.tw/news/aipl/201807130025.aspx 